\title{
POSITIVE SOLUTIONS FOR VECTOR DIFFERENTIAL EQUATIONS
}

\author{
YAN WANG \\ (Communicated by Yingfei Yi)
}

\begin{abstract}
In this paper, we are concerned with the existence and multiplicity of positive periodic solutions for first-order vector differential equations. By using the Leray-Schauder alternative theorem and the Kransnosel'skii fixed point theorem, we show that the differential equations under the periodic boundary value conditions have at least two positive periodic solutions.
\end{abstract}

\section{INTRODUCTION}

This paper is concerned with the existence and multiplicity of positive periodic solutions of the first-order non-autonomous dynamical system

$$
x^{\prime}(t)+A(t) x(t)=f(t, x) \text { for all } t \in[0, \omega]
$$

or

$$
-x^{\prime}(t)+A(t) x(t)=f(t, x) \text { for all } t \in[0, \omega],
$$

where $\omega$ is a positive constant and $f:[0, \omega] \times \mathbb{R}^{m} \rightarrow \mathbb{R}^{m}$ is a continuous function with $f(t+\omega, x)=f(t, x)$. By a $\omega$-periodic positive solution of Eq. (1.1) (or (1.2)), we mean an absolutely continuous function $x(t)=\left(x^{1}(t), x^{2}(t), \cdots, x^{m}(t)\right)^{T}$ satisfying Eq. (1.1) (or (1.2)) and the periodic boundary condition

$$
x(0)=x(\omega) .
$$

In mathematical ecology, it is found that in a particular case of Eq. (1.2), the $m$-species Lotka-Volterra competition system,

$$
\dot{y}_{i}(t)=y_{i}(t)\left[b_{i}(t)-\sum_{j=1}^{m} a_{i j}(t) y_{j}(t)\right], i=1,2, \cdots, m,
$$

where the coefficients are assumed to be continuous $\omega$-periodic functions. The assumption of the periodicity of $b_{i}$ and $a_{i j}$ for $i, j=1,2, \cdots, m$ is a way of incorporating the periodicity of the environment (e.g., seasonal effects of weather, food supplies, mating habits, harvesting, etc.). Many papers have been devoted to studying this system (see [1,4, 12,13 and the references therein).

Mathematically, the multiplicity and existence of positive periodic solutions for Eqs. (1.1) and (1.3) have been extensively studied in the literature (see [2, 3, 5, 10]).

Received by the editors December 14, 2011 and, in revised form, February 2, 2012.

2010 Mathematics Subject Classification. Primary 47H10; Secondary 34C25.

Key words and phrases. Positive solution, Leray-Schauder alternative theorem, Kransnosel'skii fixed point theorem.

This work was supported by the TianYuan Special Funds of the NNSF of China (Grant No. 11026168). 
There are three common methods used to study such problems. The first one is the method of upper and lower solutions, coupled with the Lyapunov-Schmidt method and the monotone iterative technique (see $[7,8]$ ). The second one is the coincidence degree theory of Mawhin (see [9]). The third one is the fixed point theorems, such as the Schauder fixed point theorem and the Krasonsel'skii fixed point theorem in a cone. There is a rich literature on the use of the Krasonsel'skii fixed point theorem for the existence of positive solutions of boundary value problems for differential equations (see [5, 10,11]).

Motivated by recent papers (see [2,6]), we investigate the existence and multiplicity of positive periodic solutions of the first-order vector differential equations, and we obtain several results based on the Leray-Schauder alternative theorem and the Kransnosel'skii fixed point theorem by constructing a cone defined in $\mathbb{R}^{m}$. In this paper, we are mainly interested in two cases of the non-linearity of $f(t, x)$ in (1.1). The first case is a positone, and the second case is a semi-positone. It is difficult to find the results of the semi-positone case. We show the results of the semi-positone case by the results of the positone case in this paper.

The paper is organized as follows. In section 2, we introduce some notation and preliminary results. In section 3 , some existence and multiplicity results in the positone case will be given. Finally, we study the semi-positone case in section 4 .

\section{Notation AND PRELIMINARIES}

Throughout this paper, $x^{i}$ denotes the $i$ th component of $x \in \mathbb{R}^{m},|x|=\max \left\{\left|x^{i}\right|\right.$, $i=1,2, \cdots, m\}$ denotes the norm of $x \in \mathbb{R}^{m}$, and $C_{\omega}$ denotes the Banach space of all continuous functions $x:[0, \omega] \rightarrow \mathbb{R}^{m}$ endowed with the norm $\|x\|=\sup \{|x(t)|$, $t \in[0, \omega]\}$. The notation $x \geq\left(>, \leq,<\right.$, respectively) y means that $x^{i} \geq(>, \leq,<$, respectively) $y^{i}$ for $i=1,2, \cdots, m$. The notation $x \succ 0$ denotes $x(t) \geq 0$ for all $t \in[0, \omega]$, and $x(t)>0$ for $t$ in a subset of positive measure.

Consider the first-order linear boundary value problem

$$
\begin{aligned}
x^{\prime}(t)+A(t) x & =h(t), \text { for all } t \in[0, \omega], \\
x(0) & =x(\omega),
\end{aligned}
$$

where $h \in L^{1}\left([0, \omega], \mathbb{R}^{m}\right)$ and $A(t)$ satisfies the following condition:

(A) $A(t)=\operatorname{diag}\left(a_{i}(t)\right)$, where $a_{i}(t):[0, \omega] \rightarrow \mathbb{R}$ is a continuous function with $a_{i} \succ 0, a_{i}(t+\omega)=a_{i}(t)$, for $i=1,2, \cdots, m$.

Let

$$
U(t, s)=\operatorname{diag}\left(u_{i}(t, s)\right),(t, s) \in[0, \omega] \times[0, \omega],
$$

where $u_{i}(t, s)=\frac{e^{-\int_{s}^{t} a_{i}(\tau) d \tau}}{1-e^{-\int_{0}^{\omega} a_{i}(\tau) d \tau}}, i=1,2, \cdots, m$. Obviously, $u_{i}(t, s)$ is an absolutely continuous non-negative function on $[0, \omega] \times[0, \omega]$, and satisfies

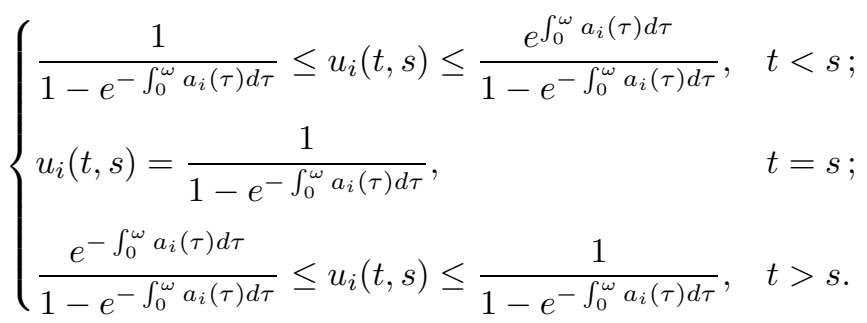

Thus, the following lemma holds. 
Lemma 2.1. Suppose that $h \in L^{1}\left([0, \omega], \mathbb{R}^{m}\right)$ and $A(t)$ satisfies condition (A). Then the boundary value problem (2.1) has a unique solution

$$
x(t)=\int_{0}^{t} U(t, s) h(s) d s+\Delta \int_{t}^{\omega} U(t, s) h(s) d s,
$$

where $\Delta=\operatorname{diag}\left(\delta_{i}\right), \delta_{i}=e^{-\int_{0}^{\omega} a_{i}(\tau) d \tau}$.

Set

$$
G(t, s)= \begin{cases}U(t, s), & t \geq s \\ \Delta U(t, s), & t<s\end{cases}
$$

Then the unique solution of (2.1) has the following form:

$$
x(t)=\int_{0}^{\omega} G(t, s) h(s) d s .
$$

Under condition (A), we denote

$$
m_{i}=\min _{0 \leq s, t \leq \omega} G_{i}(t, s), \quad M_{i}=\max _{0 \leq s, t \leq \omega} G_{i}(t, s), \quad \text { for } i=1,2, \cdots, m,
$$

where $G(t, s)=\operatorname{diag}\left(G_{i}(t, s)\right)$. Obviously, $M_{i}>m_{i}>0$ and $0<m_{i} / M_{i}=\delta_{i}<1$ for $i=1,2, \cdots, m$.

Set

$$
K=\left\{x \in C_{\omega}: x^{i}(t) \geq \delta_{i}\left\|x^{i}\right\|, i=1,2, \cdots, m, t \in[0, \omega]\right\} .
$$

Obviously, $K$ is a cone in $C_{\omega}$.

Suppose that $F:[0, \omega] \times \mathbb{R}^{m} \rightarrow \mathbb{R}_{+}^{m}$ is a continuous function. Define an operator $T: C_{\omega} \rightarrow C_{\omega}$ by

$$
T(x)(t)=\int_{0}^{\omega} G(t, s) F(s, x(s)) d s \text { for } t \in[0, \omega] .
$$

Lemma 2.2. $T$ is well defined and maps $C_{\omega}$ into $K$. Moreover, $T$ is continuous and completely continuous.

Proof. By (2.2), for any $t \in[0, \omega], x \in C_{\omega}$ and $i=1,2, \cdots, m$, we have that

$$
(T(x))^{i}(t) \leq \int_{0}^{\omega} \frac{1}{1-e^{-\int_{0}^{\omega} a_{i}(\tau) d \tau}} F^{i}(s, x(s)) d s
$$

and

$$
(T(x))^{i}(t) \geq \int_{0}^{\omega} \frac{e^{-\int_{0}^{\omega} a_{i}(\tau) d \tau}}{1-e^{-\int_{0}^{\omega} a_{i}(\tau) d \tau}} F^{i}(s, x(s)) d s \geq \delta_{i}\left\|(T(x))^{i}\right\|,
$$

where $F^{i}(t, x)$ is the $i$ th component of $F(t, x)$. Therefore, $T$ is well defined and maps $C_{\omega}$ into $K$.

Note that $u_{i}(t, s)$ and $F(t, x)$ are continuous; we can easily get the continuity of $T$. By the Arzelá-Ascoli theorem and the Lebesgue dominated convergence theorem, it is not difficult to show that $T$ is completely continuous. We omit the details here.

In the following, we recall the following well-known theorems.

Lemma 2.3 (Leray-Schauder alternative theorem). Assume $\Omega$ is a relatively compact subset of a convex set $K$ in a normed space $X$. Let $T: \bar{\Omega} \rightarrow K$ be a compact map with $0 \in \Omega$. Then one of the following two conditions holds:

(i) $T$ has at least one fixed point in $\bar{\Omega}$.

(ii) There exist $x \in \partial \Omega$ and $0<\lambda<1$ such that $x=\lambda T x$. 
Lemma 2.4 (Kransnosel'skii fixed point theorem). Let $K$ be a cone in a Banach space $X$ and $\Omega_{1}, \Omega_{2}$ be two bounded open sets in $X$ such that $0 \in \Omega_{1}$ and $\bar{\Omega}_{1} \subset \Omega_{2}$. Let $T: K \cap\left(\bar{\Omega}_{2} \backslash \Omega_{1}\right) \rightarrow K$ be a completely continuous operator such that either

(i) $\|T u\| \geq\|u\|, u \in K \cap \partial \Omega_{1}$, and $\|T u\| \leq\|u\|, u \in K \cap \partial \Omega_{2}$; or

(ii) $\|T u\| \leq\|u\|, u \in K \cap \partial \Omega_{1}$, and $\|T u\| \geq\|u\|, u \in K \cap \partial \Omega_{2}$.

Then $T$ has a fixed point in $K \cap\left(\bar{\Omega}_{2} \backslash \Omega_{1}\right)$.

\section{Positone case}

In this section, the existence and multiplicity of positive solutions for problems (1.1), (1.3) are obtained in the positone case.

Suppose that $f(t, x)$ satisfies the following conditions.

(F1) For each constant $L>0$, there exists a function $\phi_{L} \succ 0$ such that $f^{i}(t, x) \geq$ $\phi_{L}^{i}(t)$ for all $(t, x) \in[0, \omega] \times X_{L}$, where $X_{L}=(0, L] \times(0, L] \times \cdots \times(0, L] \subset$ $\mathbb{R}^{m}$, and $f^{i}(t, x)$ and $\phi_{L}^{i}(t)$ are the $i$ th components of $f(t, x)$ and $\phi_{L}$, respectively, for $i=1,2, \cdots, m$.

(F2) There exist continuous, non-negative functions $g, h: \mathbb{R}_{+}^{m} \rightarrow \mathbb{R}^{m}$ such that

$$
f(t, x) \leq g(x)+h(x) \text { for all }(t, x) \in[0, \omega] \times \mathbb{R}_{+}^{m},
$$

where $g(x)>0$ is non-increasing and $h(x) / g(x)$ is non-decreasing in $x \in$ $\mathbb{R}_{+}^{m}$.

(F3) There exists a positive number $r$ such that

$$
\left\|w^{i}\right\| g^{i}\left(\delta_{i} \tilde{r}_{i}\right)\left(1+h^{i}(r I) / g^{i}(r I)\right)<r \text { for } i=1,2, \cdots, m,
$$

where $I=(1,1, \cdots, 1)^{T} \in \mathbb{R}^{m}$, and $g^{i}(t), h^{i}(t), w^{i}(t)$ are the $i$ th components of $g(t), h(t), w(t)=\int_{0}^{\omega} G(t, s) I d s$, respectively. The notation $\tilde{r}_{i}$ denotes an $n$-dimensional vector, the $i$ th component of $\tilde{r}_{i}$ is $r$, and other components are zero.

Theorem 3.1. Suppose that $A(t)$ satisfies (A) and $f(t, x)$ satisfies (F1)-(F3). Then Eqs. (1.1), (1.3) have at least one positive periodic solution with $0<\|x\|<r$.

Proof. Let $N_{0}=\left\{n_{0}, n_{0}+1, \cdots\right\}$, where $n_{0} \in\{1,2, \cdots\}$ is chosen such that

$$
\left\|w^{i}\right\| g^{i}\left(\delta_{i} \tilde{r}_{i}\right)\left(1+h^{i}(r I) / g^{i}(r I)\right)+1 / n_{0}<r \text { for } i=1,2, \cdots, m .
$$

Fix $n \in N_{0}$. Consider the family of equations

$$
x^{\prime}(t)+A(t) x(t)=\lambda f_{n}(t, x)+A(t) I / n,(t, x) \in[0, \omega] \times \mathbb{R}^{m},
$$

where $\lambda \in[0,1]$ and $f_{n}^{i}(t, x)=f^{i}\left(t, \bar{x}_{i}\right)$ with $\bar{x}_{i}=\left(x^{1}, \cdots, x^{i-1}, \max \left\{x^{i}, 1 / n\right\}, x^{i+1}\right.$, $\left.\cdots, x^{n}\right)^{T}$ for $i=1,2, \cdots, m$. The boundary value problems (3.1), (1.3) are equivalent to the following fixed point problem in $C_{\omega}$ :

$$
\begin{aligned}
x(t) & =\int_{0}^{\omega} G(t, s) \lambda f_{n}(s, x(s)) d s+1 / n \int_{0}^{\omega} G(t, s) A(s) I d s \\
& =\lambda T_{n} x+1 / n \int_{0}^{\omega} G(t, s) A(s) I d s,
\end{aligned}
$$

where $T_{n}$ denotes the operator defined by (2.3), with $F(t, x)$ replaced by $f_{n}(t, x)$. 
Since

$$
\begin{aligned}
\left(1 / n \int_{0}^{\omega} G(t, s) A(s) I d s\right)^{i}= & \frac{1}{n}\left(\int_{0}^{t} u_{i}(t, s) a_{i}(s) d s+\delta_{i} \int_{t}^{\omega} u_{i}(t, s) a_{i}(s) d s\right) \\
= & \frac{1}{n\left(1-e^{-\int_{0}^{\omega} a_{i}(\tau) d \tau}\right)}\left(\int_{0}^{t} e^{-\int_{s}^{t} a_{i}(\tau) d \tau} a_{i}(s) d s\right. \\
& \left.+\delta_{i} \int_{t}^{\omega} e^{-\int_{s}^{t} a_{i}(\tau) d \tau} a_{i}(s) d s\right),
\end{aligned}
$$

and noting that

we obtain

$$
a_{i}(s) e^{-\int_{s}^{t} a_{i}(\tau) d \tau}=\frac{d}{d s}\left(e^{-\int_{s}^{t} a_{i}(\tau) d \tau}\right)
$$

$$
\begin{aligned}
\left(1 / n \int_{0}^{\omega} G(t, s) A(s) I d s\right)^{i} & =\frac{1-e^{-\int_{0}^{t} a_{i}(\tau) d \tau}+\delta_{i}\left(e^{-\int_{\omega}^{t} a_{i}(\tau) d \tau}-1\right)}{n\left(1-e^{-\int_{0}^{\omega} a_{i}(\tau) d \tau}\right)} \\
& =1 / n,
\end{aligned}
$$

for $i=1,2, \cdots, m$. Thus the fixed problem (3.2) is equivalent to the following:

$$
x=\lambda T_{n}(x)+1 / n I,
$$

i.e.

$$
x^{i}=\lambda\left(T_{n}(x)\right)^{i}+1 / n .
$$

Suppose that $x$ is a fixed point of (3.3) for some $\lambda \in[0,1]$; then $\|x\| \neq r$. Otherwise, there exists $\lambda_{0} \in[0,1]$ such that $\|x\|=r$; i.e., there exists $i_{0} \in\{1,2, \cdots, m\}$ such that $\sup _{t \in[0, \omega]}\left|x^{i_{0}}(t)\right|=r$. Note (3.3) and $f_{n}(t, x) \geq 0$; then by Lemma 2.2 we have

$$
\begin{aligned}
x^{i}(t) & \geq 1 / n, \\
x^{i}(t)-1 / n=\lambda\left(T_{n}(x)\right)^{i}(t) & \geq \delta_{i}\left\|\lambda\left(T_{n}(x)\right)^{i}(t)\right\|=\delta_{i}\left\|x^{i}-1 / n\right\|,
\end{aligned}
$$

for all $t \in[0, \omega], \lambda \in[0,1]$ and $i=1,2, \cdots, m$.

Hence, for all $t \in[0, \omega]$, we have

$$
r \geq x^{i_{0}}(t) \geq 1 / n+\delta_{i_{0}}\left\|x^{i_{0}}-1 / n\right\| \geq 1 / n+\delta_{i_{0}}(r-1 / n)>\delta_{i_{0}} r .
$$

According to (F2), (3.5), and the choice of $n_{0}$ with $1 / n<1 / n_{0}<r$, for all $t \in[0, \omega]$,

$$
\begin{aligned}
x^{i_{0}}(t) & =\left(\lambda \int_{0}^{\omega} G(t, s) f_{n}(s, x(s)) d s\right)^{i_{0}}+1 / n \\
& \leq\left(\int_{0}^{\omega} G(t, s) f(s, x(s)) d s\right)^{i_{0}}+1 / n \\
& \leq \int_{0}^{\omega} G_{i_{0}}(t, s) g^{i_{0}}(x(s))\left(1+h^{i_{0}}(x(s)) / g^{i_{0}}(x(s))\right) d s+1 / n \\
& \leq g^{i_{0}}\left(\delta_{i_{0}} \tilde{r}_{i_{0}}\right)\left(1+h^{i_{0}}(r I) / g^{i_{0}}(r I)\right) \int_{0}^{\omega} G_{i_{0}}(t, s) d s+1 / n_{0} .
\end{aligned}
$$

Thus,

$$
r=\|x\| \leq\left\|w^{i_{0}}\right\|\left(g^{i_{0}}\left(\delta_{i_{0}} \tilde{r}_{i_{0}}\right)\left(1+h^{i_{0}}(r I) / g^{i_{0}}(r I)\right)\right)+1 / n_{0},
$$

which is a contradiction to the choice of $n_{0}$. 
By the Leray-Schauder alternative theorem, (3.3) (with $\lambda=1$ ) admits a fixed point $x_{n} \in B_{r}=\left\{x \in \mathbb{R}^{m}:\|x\|<r\right\}$. That is, $x_{n}$ is the periodic solution of (3.1), (1.3) (with $\lambda=1$ ) with $\left\|x_{n}\right\|<r$. Note $x_{n}(t) \geq 1 / n I$ by (3.3) (with $\lambda=1$ ) for all $t \in[0, \omega]$; therefore $x_{n}$ is actually a positive solution of (3.1), (1.3) (with $\lambda=1$ ).

In the following, we will show that $x_{n}$ has a uniform positive lower bounded; i.e., there exists a constant $\theta \in \mathbb{R}_{+}^{m}$, independent of $n \in N_{0}$ such that

$$
\min _{t} x_{n}(t) \geq \theta
$$

for all $n \in N_{0}$.

From (F1), there exists a function $\phi_{r} \succ 0$ such that $f^{i}(t, x) \geq \phi_{r}^{i}(t)$ for $(t, x) \in$ $[0, \omega] \times X_{r}$ and $i=1,2, \cdots, m$. Suppose that $x_{r}(t)$ is the unique periodic solution to problem (2.1) with $h=\phi_{r}$. Then

$$
x_{r}(t)=\int_{0}^{\omega} G(t, s) \phi_{r}(s) d s \geq \min _{i}\left\{m_{i}\right\} \int_{0}^{\omega} \phi_{r}(s) d s>0 .
$$

Note that

$$
\begin{aligned}
x_{n}(t) & =\int_{0}^{\omega} G(t, s) f_{n}\left(s, x_{n}(s)\right) d s+1 / n I \\
& =\int_{0}^{\omega} G(t, s) f\left(s, x_{n}(s)\right) d s+1 / n I \\
& \geq \int_{0}^{\omega} G(t, s) \phi_{r}(s) d s+1 / n I \\
& =x_{r}(t)+1 / n I \\
& \geq \min _{i}\left\{m_{i}\right\} \int_{0}^{\omega} \phi_{r}(s) d s=\theta .
\end{aligned}
$$

Since $\left\|x_{n}\right\|<r$ and $\left\{x_{n}\right\}_{n \in N_{0}}$ is a bounded and equi-continuous family on $[0, \omega]$, according to the Arzelá-Ascoli theorem, there exists a subsequence $\left\{x_{n_{k}}\right\}_{k \in \mathbb{N}}$ converging uniformly on $[0, \omega]$ to a function $x \in C_{\omega}$. By the integral equation

$$
x_{n_{k}}(t)=\int_{0}^{\omega} G(t, s) f\left(s, x_{n_{k}}(s)\right) d s+1 / n_{k} I
$$

letting $k \rightarrow \infty$, we have that

$$
x(t)=\int_{0}^{\omega} G(t, s) f(s, x(s)) d s .
$$

Since $\left\|x_{n}\right\|<r$ and by (3.6), $x$ is a positive periodic solution of (1.1), (1.3) with $0<\|x\| \leq r$.

Finally, it is not difficult to show that $\|x\|<r$ by noting that if $\|x\|=r$, the argument similar to the proof of the first claim will yield a contradiction.

Let us assume that:

(F2') For each component $f^{i}(t, x)$ of $f(t, x)$, there exist continuous, non-negative functions $g^{i}, h^{i}, k^{i}: \mathbb{R}_{+}^{m} \rightarrow \mathbb{R}$ such that

$$
f^{i}(t, x) \leq k^{i}(t)\left(g^{i}(x)+h^{i}(x)\right) \text { for all }(t, x) \in[0, \omega] \times \mathbb{R}_{+}^{m},
$$

where $g^{i}(x)>0$ is non-increasing and $h^{i}(x) / g^{i}(x)$ is non-decreasing in $x \in \mathbb{R}_{+}^{m}$ for $i=1,2, \cdots, m$. 
(F3') There exists a positive number $r$ such that

$$
\left\|\left(w^{*}\right)^{i}\right\| g^{i}\left(\delta_{i} \tilde{r}_{i}\right)\left(1+h^{i}(r I) / g^{i}(r I)\right)<r \text { for } i=1,2, \cdots, m,
$$

where $\left(w^{*}\right)^{i}=\int_{0}^{\omega} G_{i}(t, s) k^{i}(s) d s$.

We have the following result.

Theorem 3.2. Suppose that $A(t)$ satisfies (A) and $f(t, x)$ satisfies (F1), (F2')(F3'). Then Eqs. (1.1), (1.3) have at least one positive periodic solution with $0<\|x\|<r$.

Proof. The proof is similar to the proof of Theorem 3.1, so we omit the details here.

Theorem 3.3. Suppose that (A) and (F1)-(F3) hold. Furthermore, assume that:

(F5) There exist continuous, non-negative functions $g_{1}, h_{1}: \mathbb{R}_{+}^{m} \rightarrow \mathbb{R}^{m}$ such that

$$
f(t, x) \geq g_{1}(x)+h_{1}(x) \text { for all }(t, x) \in[0, \omega] \times \mathbb{R}_{+}^{m},
$$

where $g_{1}(x)>0$ is non-increasing and $h_{1}(x) / g_{1}(x)$ is non-decreasing in $x \in \mathbb{R}_{+}^{m}$.

(F6) There exists a positive number $R>r$ such that

$$
g_{1}^{i}(R I)\left(1+h_{1}^{i}\left(\delta_{j} \tilde{R}_{j}\right) / g_{1}^{i}\left(\delta_{j} \tilde{R}_{j}\right)\right)\left\|w^{i}\right\| \geq R, \text { for } i, j=1,2, \cdots, m,
$$

where $\tilde{R}_{j}$ denotes an $m$-dimensional vector, the $j$ th component of $\tilde{R}_{j}$ is $R$, and other components are zero. Also, $g_{1}^{i}, h_{1}^{i}$ denote the ith components of $g_{1}, h_{1}$, respectively.

Then, besides the periodic solution $x$ constructed in Theorem 3.1, the boundary value problems (1.1), (1.3) have another positive periodic solution $\tilde{x}$ with $r<\|\tilde{x}\| \leq$ $R$.

Proof. Let $\Omega_{1}=B_{r}$ and $\Omega_{2}=B_{R}$ be balls in $C_{\omega}$. The operator $T: K \cap\left(\bar{\Omega}_{2} \backslash \Omega_{1}\right) \rightarrow$ $K$ is defined by (2.3), with $F(t, x)$ replaced by $f(t, x)$. The operator is well defined on $K \cap\left(\bar{\Omega}_{2} \backslash \Omega_{1}\right)$.

First we claim that $\|T x\|<\|x\|$ for $x \in K \cap \partial \Omega_{1}$. In fact, if $x \in K \cap \partial \Omega_{1}$, we have $\|x\|=r$. As in the proof of $\left\|x_{n}\right\|<r$ in Theorem 3.1, we obtain $\|T x\|<r$. We omit the details.

Next we show that $\|T x\| \geq\|x\|$ for $x \in K \cap \partial \Omega_{2}$. If $x \in K \cap \partial \Omega_{2}$, then $\|x\|=R$; i.e., there exists an $i_{0} \in\{1,2, \cdots, m\}$ such that $\sup _{t \in[0, \omega]}\left|x^{i_{0}}(t)\right|=R$ and $x^{i_{0}}(t) \geq \delta_{i_{0}} R$. Thus, for $i=1,2, \cdots, m$, we have that

$$
\begin{aligned}
(T(x)(t))^{i} & =\left(\int_{0}^{\omega} G(t, s) f(s, x(s)) d s\right)^{i} \\
& \geq \int_{0}^{\omega} G_{i}(t, s) g_{1}^{i}(x(s))\left(1+h_{1}^{i}(x(s)) / g_{1}^{i}(x(s))\right) d s \\
& \geq \int_{0}^{\omega} G_{i}(t, s) g_{1}^{i}(R I)\left(1+h_{1}^{i}\left(\delta_{i_{0}} \tilde{R}_{i_{0}}\right) / g_{1}^{i}\left(\delta_{i_{0}} \tilde{R}_{i_{0}}\right)\right) d s \\
& \geq g_{1}^{i}(R I)\left(1+h_{1}^{i}\left(\delta_{i_{0}} \tilde{R}_{i_{0}}\right) / g_{1}^{i}\left(\delta_{i_{0}} \tilde{R}_{i_{0}}\right)\right) w^{i}(t) .
\end{aligned}
$$

Therefore,

$$
\|T x\| \geq\left\|(T x)^{i}\right\| \geq g_{1}^{i}(R I)\left(1+h_{1}^{i}\left(\delta_{i_{0}} \tilde{R}_{i_{0}}\right) / g_{1}^{i}\left(\delta_{i_{0}} \tilde{R}_{i_{0}}\right)\right)\left\|w^{i}\right\| \geq R=\|x\| .
$$


By Lemma 2.4, T has a fixed point $\tilde{x} \in K \cap\left(\bar{\Omega}_{2} \backslash \Omega_{1}\right)$. Obviously, $\tilde{x}$ is a positive periodic solution of (1.1), (1.3) and satisfies $r<\|\tilde{x}\| \leq R$.

Weakening the assumptions (F5) and (F6), we have the following result.

Theorem 3.4. Suppose that (A) and (F1), (F2') and (F3') hold. Furthermore, assume that

(F5') For each component $f^{i}$ of $f$, there exist continuous, non-negative functions $g_{1}^{i}, h_{1}^{i}, k_{1}^{i}: \mathbb{R}_{+}^{m} \rightarrow \mathbb{R}$ such that

$$
f^{i}(t, x) \geq k_{1}^{i}(t)\left(g_{1}^{i}(x)+h_{1}^{i}(x)\right) \text { for all }(t, x) \in[0,1] \times \mathbb{R}_{+}^{m},
$$

where $g_{1}^{i}(x)>0$ is non-increasing and $h_{1}^{i}(x) / g_{1}^{i}(x)$ is non-decreasing in $x \in \mathbb{R}_{+}^{m}$ for $i=1,2, \cdots, m$.

(F6') There exists a positive number $R>r$ such that

$$
g_{1}^{i}(R I)\left(1+h_{1}^{i}\left(\delta_{j} \tilde{R}_{j}\right) / g_{1}^{i}\left(\delta_{j} \tilde{R}_{j}\right)\right)\left\|\left(w_{1}^{*}\right)^{i}\right\| \geq R \text { for } i, j=1,2, \cdots, m,
$$

where $w_{1}^{*}=\int_{0}^{\omega} G(t, s) k_{1}(s) d s$.

Then, besides the periodic solution $x$ constructed in Theorem 3.2, the boundary value problems (1.1), (1.3) have another positive periodic solution $\tilde{x}$ with $r<\|\tilde{x}\| \leq$ $R$.

\section{Semi-Positone CASE}

In this section, we consider the semi-positone case of (1.1), which means that $f(t, x)$ satisfies the following:

(F7) There exists a function $p \succ 0$ on $[0, \omega]$ with $P(t)=\int_{0}^{\omega} G(t, s) p(s) d s$ bounded such that $F(t, x):=f(t, x)+p(t) \geq 0$ for all $(t, x) \in[0, \omega] \times \mathbb{R}_{+}^{m}$.

Suppose that $A(t)$ satisfies (A) and $f(t, x)$ satisfies (F7). Furthermore, assume that

(F8) For each component $F^{i}$ of $F$, there exist continuous, non-negative functions $g^{i}, h^{i}: \mathbb{R}_{+}^{m} \rightarrow \mathbb{R}$ such that

$$
F^{i}(t, x) \leq g^{i}(x)+h^{i}(x) \text { for all }(t, x) \in[0, \omega] \times \mathbb{R}_{+}^{m},
$$

where $g^{i}(x)>0$ is non-increasing and $h^{i}(x) / g^{i}(x)$ is non-decreasing in $x \in \mathbb{R}_{+}^{m}$ for $i=1,2, \cdots, m$.

(F9) There exist a positive constant $L$ and a non-increasing positive function $\phi_{L}$ such that

$$
F^{i}(t, x) \geq \phi_{L}^{i}(x) \text { for all }(t, x) \in[0, \omega] \times X_{L} \text { and } i=1,2, \cdots, m,
$$

where $\phi_{L}^{i}$ satisfies $\lim _{x \rightarrow 0+} \phi_{L}^{i}(x)=\infty$ and $\lim _{x \rightarrow 0+} \int_{x}^{L} \phi_{L}^{i}(u I) d u=\infty$.

(F10) There exists $r>\|P\| / \delta$, where $\delta=\min _{i}\left\{\delta_{i}\right\}$, such that

$$
g^{i}\left(\delta_{i} \tilde{r}_{i}-\tilde{P}_{i}\right)\left(1+h^{i}(r I) / g^{i}(r I)\right)\left\|w^{i}\right\|<r \text { for } i=1,2, \cdots, m,
$$

where $\tilde{P}_{i} \in \mathbb{R}^{m}$ satisfies that the $i$ th component is $\left\|P^{i}\right\|$ with $P^{i}(t)$ denoting the $i$ th component of $P(t)$ and the other components of $\tilde{P}_{i}$ are zero. The notation $\tilde{r}_{i}$ is similar to that in (F3). 
Consider the following vector differential equation:

$$
x^{\prime}(t)+A(t) x(t)=F(t, x(t)-P(t)) .
$$

If (4.1), (1.3) have a solution $x$ that satisfies $x(t)>P(t)$ for all $t \in[0, \omega], u(t)=$ $x(t)-P(t)$ is a positive solution of (1.1), (1.3).

As in the proof of Theorem 3.1, set $N_{0}=\left\{n_{0}, n_{0}+1, \cdots\right\}$, where $n_{0} \in\{1,2, \cdots\}$ is chosen such that

$$
g^{i}\left(\delta_{i} \tilde{r}_{i}-\tilde{P}_{i}\right)\left(1+h^{i}(r I) / g^{i}(r I)\right)\left\|w^{i}\right\|+1 / n_{0}<r .
$$

Fix $n \in N_{0}$. Consider the family of equations

$$
x^{\prime}(t)+A(t) x(t)=\lambda F_{n}(t, x(t)-P(t))+A(t) I / n,(t, x) \in[0, \omega] \times \mathbb{R}^{m},
$$

where $\lambda \in[0,1]$ and

$$
F_{n}^{i}(t, x)= \begin{cases}F^{i}(t, x), & \text { if } x^{i} \geq 1 / n, \\ F^{i}\left(t, x^{1}, \cdots, x^{i-1}, 1 / n, x^{i+1}, \cdots, x^{n}\right), & \text { if } x^{i} \leq 1 / n .\end{cases}
$$

The boundary value problems (4.2), (1.3) are equivalent to the following fixed point problem:

$$
x(t)=\lambda \int_{0}^{\omega} G(t, s) F_{n}(s, x(s)-P(s)) d s+1 / n I .
$$

Similar to the proof of Theorem 3.1, it is not difficult to prove that $\|x\| \neq r$.

By the Leray-Schauder alternative theorem, (4.3) (with $\lambda=1$ ) admits a fixed point $x_{n} \in B_{r}$. That is, $x_{n}$ is the periodic solution of (4.2), (1.3) (with $\lambda=1$ ) with $\left\|x_{n}\right\|<r$.

To prove that (4.1), (1.3) (with $\lambda=1$ ) have a solution $x$ that satisfies $x(t)>P(t)$ for all $t \in[0, \omega]$, we give the following lemma.

Lemma 4.1. Suppose that $A(t)$ satisfies (A) and that $f(t, x)$ satisfies (F7) and (F9). Then there exist a constant $\Theta \in \mathbb{R}_{+}^{m}$ and an integer $n_{2}>n_{0}$ such that any solution $x_{n}$ of $E q$. (4.3) (with $\lambda=1$ ) satisfies

$$
x_{n}(t)-P(t) \geq \Theta \text { for all } t \in[0, \omega] \text { and } n \geq n_{2} .
$$

Proof. By condition (F9), there exist $L_{1} \in(0, L)$ and a continuous function $\phi_{L_{1}}^{i}$ satisfying (F9) such that

$$
\begin{array}{r}
F^{i}(t, x)-a_{i}(t) x^{i} \geq \phi_{L_{1}}^{i}(x)>\max \left\{\left\|p^{i}\right\|, r\left\|a_{i}\right\|_{1}\right\} \text { for }(t, x) \in[0, \omega] \times X_{L_{1}} \\
\text { and } i=1,2, \cdots, m
\end{array}
$$

where $p^{i}(t)$ is the $i$ th component of $p(t)$ and $\|\cdot\|_{1}$ denotes the $L^{1}$-norm over $(0, \omega)$.

Choose $n_{1} \in N_{0}$ such that $1 / n_{1} \leq L_{1}$, and set $N_{1}=\left\{n_{1}, n_{1}+1, \cdots\right\}$. For $i=1,2, \cdots, m$ and $n \in N_{1}$, let

$$
\alpha_{n}^{i}=\min _{0 \leq t \leq \omega}\left[x_{n}^{i}(t)-P^{i}(t)\right] \text { and } \beta_{n}^{i}=\max _{0 \leq t \leq \omega}\left[x_{n}^{i}(t)-P^{i}(t)\right] .
$$


To verify (4.4), we need the following steps:

(a) $\beta_{n}=\min _{i}\left\{\beta_{n}^{i}\right\}>L_{1}$ for all $n \in N_{1}$.

(b) There is a constant $\theta>0$ such that $\alpha_{n}=\min _{i}\left\{\alpha_{n}^{i}\right\}>\theta$.

First, we show that claim (a) holds. If not, there exists $n \in N_{1}$ such that $\beta_{n}^{i} \leq L_{1}$ for all $i=1,2, \cdots, m$. Then

$$
F_{n}^{i}\left(t, x_{n}(t)-P(t)\right)>r\left\|a_{i}\right\|_{1} .
$$

In fact, if $1 / n \leq x_{n}^{i}(t)-P^{i}(t) \leq L_{1}$ for some $i \in\{1,2, \cdots, m\}$, by (4.5) we have that

$$
\begin{aligned}
F_{n}^{i}\left(t, x_{n}(t)-P(t)\right) & =F^{i}\left(t, x_{n}(t)-P(t)\right) \\
& \geq a_{i}(t)\left(x_{n}^{i}(t)-P^{i}(t)\right)+\phi_{L_{1}}^{i}\left(x_{n}(t)-P(t)\right) \\
& \geq \phi_{L_{1}}^{i}\left(x_{n}(t)-P(t)\right) \\
& \geq r\left\|a_{i}\right\|_{1} .
\end{aligned}
$$

If $x_{n}^{i}(t)-P^{i}(t) \leq 1 / n$ for some $i \in\{1,2, \cdots, m\}$, we have that

$$
\begin{aligned}
F_{n}^{i}\left(t, x_{n}(t)-P(t)\right) & =F^{i}\left(t, x_{n} \widetilde{(t)-P}(t)\right) \\
& \geq a_{i}(t) / n+\widetilde{\phi_{L_{1}}^{i}}\left(x_{n}(\widetilde{t)-P}(t))\right. \\
& \geq \phi_{L_{1}}^{i}\left(x_{n}(\widetilde{t)-P}(t))\right. \\
& >r\left\|a_{i}\right\|_{1},
\end{aligned}
$$

where $x_{n}\left(\frac{t)-P}{t}(t)=\left(x_{n}^{1}(t)-P^{1}(t), \cdots, x_{n}^{i-1}(t)-P^{i-1}(t), 1 / n, x_{n}^{i+1}(t)-P^{i+1}(t)\right.\right.$, $\left.\cdots, x_{n}^{m}(t)-P^{m}(t)\right)^{T}$. Therefore, (4.6) holds.

For each $i=1,2, \cdots, m$, integrating Eq. (4.2) (with $\lambda=1$ ) from 0 to $\omega$, we get

$$
\begin{aligned}
0 & =\int_{0}^{\omega}\left[\left(x_{n}^{i}(t)\right)^{\prime}+a_{i}(t) x_{n}^{i}(t)-F_{n}^{i}\left(t, x_{n}(t)-P(t)\right)-a_{i}(t) / n\right] d t \\
& =\int_{0}^{\omega}\left(x_{n}^{i}(t)\right)^{\prime} d t+\int_{0}^{\omega} a_{i}(t) x_{n}^{i}(t) d t-\int_{0}^{\omega} F_{n}^{i}\left(t, x_{n}(t)-P(t)\right) d t-\int_{0}^{\omega} a^{i}(t) / n d t \\
& =x_{n}^{i}(\omega)-x_{n}^{i}(0)+\int_{0}^{\omega} a_{i}(t) x_{n}^{i}(t) d t-\int_{0}^{\omega} F_{n}^{i}\left(t, x_{n}(t)-P(t)\right) d t-\int_{0}^{\omega} a_{i}(t) / n d t \\
& <\int_{0}^{\omega} a_{i}(t) x_{n}^{i}(t) d t-\int_{0}^{\omega} F_{n}^{i}\left(t, x_{n}(t)-P(t)\right) d t \\
& \leq r \int_{0}^{\omega} a_{i}(t) d t-r\left\|a_{i}\right\|_{1}=0 .
\end{aligned}
$$

This is a contradiction. Therefore,

$$
\beta_{n}^{i}>L_{1} \text { for all } n \in N_{1} \text { and } i=1,2, \cdots, m .
$$

In the following, we will prove step (b). There are two cases:

(b1) $\alpha_{n} \geq L_{1}$.

(b2) $\alpha_{n}<L_{1}$.

For case (b1), it is not difficult to obtain (4.4). If (b2) holds, there exist $c_{n} \in[0, \omega]$ and some $i_{0} \in\{1,2, \cdots, m\}$ such that

$$
\alpha_{n}=\alpha_{n}^{i_{0}}=\min _{0 \leq t \leq \omega}\left[x_{n}^{i_{0}}(t)-P^{i_{0}}(t)\right]=\left[x_{n}^{i_{0}}\left(c_{n}\right)-P^{i_{0}}\left(c_{n}\right)\right]<L_{1} .
$$


According to (4.7), there exists $d_{n} \in[0, \omega]$ such that $x_{n}^{i_{0}}\left(d_{n}\right)-P^{i_{0}}\left(d_{n}\right)=L_{1}$. Without loss of generality, we assume $c_{n}<d_{n}$ and $x_{n}^{i_{0}}(t)-P^{i_{0}}(t) \leq L_{1}$ for $t \in$ $\left[c_{n}, d_{n}\right]$. Then

$$
F_{n}^{i_{0}}\left(t, x_{n}(t)-P(t)\right)>a_{i_{0}}(t)\left(x_{n}^{i}(t)-P^{i_{0}}(t)\right)+\left\|p^{i_{0}}\right\| \text { for } t \in\left[c_{n}, d_{n}\right] .
$$

In fact, if $1 / n \leq x_{n}^{i_{0}}(t)-P^{i_{0}}(t) \leq L_{1}$ for $t \in\left[c_{n}, d_{n}\right]$, then

$$
\begin{aligned}
F_{n}^{i_{0}}\left(t, x_{n}(t)-P(t)\right) & =F^{i_{0}}\left(t, x_{n}(t)-P(t)\right) \\
& \geq a_{i_{0}}(t)\left(x_{n}^{i_{0}}(t)-P^{i_{0}}(t)\right)+\phi_{L_{1}}^{i_{0}}\left(x_{n}(t)-P(t)\right) \\
& >a_{i_{0}}(t)\left(x_{n}^{i_{0}}(t)-P^{i_{0}}(t)\right)+\left\|p^{i_{0}}\right\| .
\end{aligned}
$$

If $x_{n}^{i_{0}}(t)-P^{i_{0}}(t)<1 / n$ for $t \in\left[c_{n}, d_{n}\right]$, then

$$
\begin{aligned}
F_{n}^{i_{0}}\left(t, x_{n}(t)-P(t)\right) & =F^{i_{0}}\left(t, x_{n}(\widetilde{t)-P}(t))\right. \\
& \geq a_{i_{0}}(t) 1 / n+\phi_{L_{1}}^{i_{0}}\left(x_{n}(\widetilde{t)-P}(t))\right. \\
& >a_{i_{0}}(t)\left(x_{n}^{i_{0}}(t)-P^{i_{0}}(t)\right)+\left\|p^{i_{0}}\right\| .
\end{aligned}
$$

Thus, 4.8 holds.

Since

$$
P(t)=\int_{0}^{\omega} G(t, s) p(s) d s,
$$

this implies that $P(t)$ is the solution of the boundary value problem

$$
\begin{aligned}
x^{\prime}(t)+A(t) x & =p(t), \\
x(0) & =x(\omega) .
\end{aligned}
$$

Thus,

$$
\left(P^{i_{0}}(t)\right)^{\prime}=p^{i_{0}}(t)-a_{i_{0}}(t) P^{i_{0}}(t)
$$

and then

$$
\begin{aligned}
\left(x_{n}^{i_{0}}(t)\right)^{\prime}-\left(P^{i_{0}}(t)\right)^{\prime}= & -a_{i_{0}}(t) x_{n}^{i_{0}}(t)+F_{n}^{i_{0}}\left(t, x_{n}(t)-P(t)\right)+a_{i_{0}}(t) / n \\
& -p^{i_{0}}(t)+a_{i_{0}}(t) P^{i_{0}}(t) \\
>\quad & -a_{i_{0}}(t) x_{n}^{i_{0}}(t)+a_{i_{0}}(t)\left(x_{n}^{i_{0}}(t)-P^{i_{0}}(t)\right) \\
& +\left\|p^{i_{0}}\right\|+a_{i_{0}}(t) / n-p^{i_{0}}(t)+a_{i_{0}}(t) P^{i_{0}}(t) \\
> & a_{i_{0}}(t) / n>0 \text { for } t \in\left[c_{n}, d_{n}\right],
\end{aligned}
$$

which implies that $y_{n}^{i_{0}}:=x_{n}^{i_{0}}-P^{i_{0}}$ is strictly increasing on $\left[c_{n}, d_{n}\right]$.

In the following, we will show that

$$
x_{n}^{i_{0}}(t)-P^{i_{0}}(t) \geq 1 / n .
$$

Otherwise, $\alpha_{n}<1 / n$ for some $n \in N_{1}$. Then there exists $e_{n} \in\left(c_{n}, d_{n}\right)$ such that $x_{n}^{i_{0}}\left(e_{n}\right)-P^{i_{0}}\left(e_{n}\right)=1 / n$ and

$$
\begin{aligned}
x_{n}^{i_{0}}(t)-P^{i_{0}}(t) \leq 1 / n & \text { for } c_{n} \leq t \leq e_{n}, \\
1 / n \leq x_{n}^{i_{0}}(t)-P^{i_{0}}(t) \leq L_{1} & \text { for } e_{n} \leq t \leq d_{n} .
\end{aligned}
$$


Note that

$$
\begin{aligned}
& \int_{1 / n}^{L_{1}} F^{i_{0}}\left(\xi_{n}^{i_{0}}\left(y_{n}^{i_{0}}\right), y_{n}^{i_{0}} I\right) d y_{n}^{i_{0}} \\
& =\int_{e_{n}}^{d_{n}} F^{i_{0}}\left(t, x_{n}^{i_{0}}(t) I-P^{i_{0}}(t) I\right)\left(\left(x_{n}^{i_{0}}(t)\right)^{\prime}-\left(P^{i_{0}}(t)\right)^{\prime}\right) d t \\
& =\int_{e_{n}}^{d_{n}} F_{n}^{i_{0}}\left(t, x_{n}^{i_{0}}(t) I-P^{i_{0}}(t) I\right)\left(\left(x_{n}^{i_{0}}(t)\right)^{\prime}-\left(P^{i_{0}}(t)\right)^{\prime}\right) d t \\
& =\int_{e_{n}}^{d_{n}}\left(\left(x_{n}^{i_{0}}(t)\right)^{\prime}+a_{i_{0}}(t) x_{n}^{i_{0}}(t)-a_{i_{0}}(t) / n\right)\left(\left(x_{n}^{i_{0}}(t)\right)^{\prime}-\left(P^{i_{0}}(t)\right)^{\prime}\right) d t \\
& =\int_{e_{n}}^{d_{n}}\left(x_{n}^{i_{0}}(t)\right)^{\prime}\left(x_{n}^{i_{0}}(t)\right)^{\prime} d t \\
& \quad+\int_{e_{n}}^{d_{n}}\left(x_{n}^{i_{0}}(t)\right)^{\prime}\left(\left(P^{i_{0}}(t)\right)^{\prime}+a_{i_{0}}(t) x_{n}^{i_{0}}(t)-a_{i_{0}}(t) / n\right) d t \\
& \quad+\int_{e_{n}}^{d_{n}}\left(a_{i_{0}}(t) / n-a_{i_{0}}(t) x_{n}^{i_{0}}(t)\right)\left(P^{i_{0}}(t)\right)^{\prime} d t,
\end{aligned}
$$

where $\xi_{n}^{i_{0}}$ denotes the inverse of $y_{n}^{i_{0}}$ restricted to $\left[c_{n}, d_{n}\right]$. The boundedness of the first term follows from the integrability of $x_{n}^{i_{0}}(t)$. Using (4.9) and $\left\|x_{n}\right\|<r$, it is not difficult to show that the second term and the last term are bounded. Thus, there exists $Q>0$ such that

$$
\int_{1 / n}^{L_{1}} F^{i_{0}}\left(\xi_{n}^{i_{0}}\left(y_{n}^{i_{0}}\right), y_{n}^{i_{0}} I\right) d y_{n}^{i_{0}}<Q .
$$

On the other hand, there exists $n_{2} \in N_{1}$ such that

$$
\int_{1 / n}^{L_{1}} F^{i_{0}}\left(\xi_{n}^{i_{0}}\left(y_{n}^{i_{0}}\right), y_{n}^{i_{0}} I\right) d y_{n}^{i_{0}} \geq \int_{1 / n}^{L_{1}} \phi_{L_{1}}^{i_{0}}(y I) d y>Q \text { for } n \in N_{2}=\left\{n_{2}, n_{2}+1, \cdots\right\} .
$$

This is a contradiction. Thus (4.10) holds.

Therefore,

$$
\begin{aligned}
& \int_{\alpha_{n}}^{L_{1}} F^{i_{0}}\left(\xi_{n}^{i_{0}}\left(y_{n}^{i_{0}}\right), y_{n}^{i_{0}} I\right) d y_{n}^{i_{0}} \\
& =\int_{c_{n}}^{d_{n}} F^{i_{0}}\left(t, x_{n}^{i_{0}}(t) I-P^{i_{0}}(t) I\right)\left(\left(x_{n}^{i_{0}}(t)\right)^{\prime}-\left(P^{i_{0}}(t)\right)^{\prime}\right) d t \\
& =\int_{c_{n}}^{d_{n}} F_{n}^{i_{0}}\left(t, x_{n}^{i_{0}}(t) I-P^{i_{0}}(t) I\right)\left(\left(x_{n}^{i_{0}}(t)\right)^{\prime}-\left(P^{i_{0}}(t)\right)^{\prime}\right) d t \\
& =\int_{c_{n}}^{d_{n}}\left(\left(x_{n}^{i_{0}}(t)\right)^{\prime}+a_{i_{0}}(t) x_{n}^{i_{0}}(t)-a_{i_{0}}(t) / n\right)\left(\left(x_{n}^{i_{0}}(t)\right)^{\prime}-\left(P^{i_{0}}(t)\right)^{\prime}\right) d t .
\end{aligned}
$$

Similar to the proof of (4.11), we can obtain that $\int_{\alpha_{n}}^{L_{1}} F^{i_{0}}\left(\xi_{n}^{i_{0}}\left(y_{n}^{i_{0}} I\right), y_{n}^{i_{0}} I\right) d y_{n}^{i_{0}}$ is bounded. On the other hand, as $n \in N_{2}$, by (F9),

$$
\int_{\alpha_{n}}^{L_{1}} F^{i_{0}}\left(\xi_{n}^{i_{0}}\left(y_{n}^{i_{0}} I\right), y_{n}^{i_{0}} I\right) d y_{n}^{i_{0}} \geq \int_{\alpha_{n}}^{L_{1}} \phi_{L}^{i_{0}}\left(y_{n}^{i_{0}} I\right) d y_{n}^{i_{0}}+Q\left(L_{1}-\alpha_{n}\right) \rightarrow \infty,
$$

as $\alpha_{n} \rightarrow 0+$. This is a contradiction. Thus (4.4) holds. 
Theorem 4.2. Suppose that $A(t)$ satisfies (A) and $f(t, x)$ satisfies (F7)-(F10). Then the boundary value problems (1.1), (1.3) have at least one positive periodic solution with $0<\|P+x\|<r$.

Proof. By Lemma 4.1 and the approach of Theorem 3.1, we can obtain the result.

Theorem 4.3. Suppose that $A(t)$ satisfies (A) and $f(t, x)$ satisfies (F7)-(F10). Furthermore, we suppose that

(F11) There exist continuous, non-negative functions $g_{1}, h_{1}: \mathbb{R}_{+}^{m} \rightarrow \mathbb{R}^{m}$ such that

$$
F(t, x) \geq g_{1}(x)+h_{1}(x) \text { for all }(t, x) \in[0, \omega] \times \mathbb{R}_{+}^{m},
$$

where $g_{1}(x)>0$ is non-increasing and $h_{1}(x) / g_{1}(x)$ is non-decreasing in $x \in \mathbb{R}_{+}^{m}$.

(F12) There exists a positive number $R>r$ such that

$$
g_{1}^{i}(R I)\left(1+h_{1}^{i}\left(\delta_{j} \tilde{R}_{j}-\tilde{P}_{j}\right) / g_{1}^{i}\left(\delta_{j} \tilde{R}_{j}-\tilde{P}_{j}\right)\right)\left\|w^{i}\right\| \geq R, \text { for } i, j=1,2, \cdots, m,
$$

where $\tilde{R}_{j}$ is as in (F6).

Then, besides the periodic solution $x$ constructed in Theorem 4.2, the boundary value problems (1.1), (1.3) have another positive periodic solution $\tilde{x}$ with $r<$ $\|\tilde{x}+P\| \leq R$.

Proof. The proof is similar to the proof of Theorem 3.3, so we omit the details here.

Similar to Theorems 3.2 and 3.4, we have the following theorems.

Theorem 4.4. Suppose that $A(t)$ satisfies (A) and $f$ satisfies (F7), (F9). Moreover, we assume that $f$ satisfies

(F8') For each component $F^{i}$ of $F$, there exist continuous, non-negative functions $g^{i}, h^{i}, k^{i}: \mathbb{R}_{+}^{m} \rightarrow \mathbb{R}$ such that

$$
F^{i}(t, x) \leq k^{i}(t)\left(g^{i}(x)+h^{i}(x)\right) \text { for all }(t, x) \in[0,1] \times \mathbb{R}_{+}^{m},
$$

where $g^{i}(x)>0$ is non-increasing and $h^{i}(x) / g^{i}(x)$ is non-decreasing in $x \in \mathbb{R}_{+}^{m}$ for $i=1,2, \cdots, m$.

(F10') There exists $r>\|P\|\|w\| / \delta$, where $\delta=\min _{i}\left\{\delta_{i}\right\}$, such that

$$
g^{i}\left(\delta_{i} \tilde{r}_{i}-\tilde{P}_{i}\right)\left(1+h^{i}(r I) / g^{i}(r I)\right)\left\|\left(w^{*}\right)^{i}\right\|<r \text { for } i=1,2, \cdots, m,
$$

where the definition of $\tilde{r}_{i}$ is similar to that in (F3) and where $w^{*}$ is as in (F3').

Then problems (1.1), (1.3) have at least one positive periodic solution with $0<$ $\|P+x\|<r$.

Theorem 4.5. Suppose that $A(t)$ and $f(t, x)$ satisfy the conditions in Theorem 4.4. Furthermore, we suppose that

(F11') For each component $F^{i}$ of $F$, there exist continuous, non-negative functions $g_{1}^{i}, h_{1}^{i}, k_{1}^{i}: \mathbb{R}_{+}^{m} \rightarrow \mathbb{R}$ such that

$$
F^{i}(t, x) \geq k_{1}^{i}(t)\left(g_{1}^{i}(x)+h_{1}^{i}(x)\right) \text { for all }(t, x) \in[0, \omega] \times \mathbb{R}_{+}^{m},
$$

where $g_{1}(x)>0$ is non-increasing and $h_{1}(x) / g_{1}(x)$ is non-decreasing in $x \in \mathbb{R}_{+}^{m}$. 
(F12') There exists a positive number $R>r$ such that

$$
\begin{aligned}
& g_{1}^{i}(R I)\left(1+h_{1}^{i}\left(\delta_{j} \tilde{R}_{j}-\tilde{P}_{j}\right) / g_{1}^{i}\left(\delta_{j} \tilde{R}_{j}-\tilde{P}_{j}\right)\right)\left\|\left(w_{1}^{*}\right)^{i}\right\| \geq R, \text { for } i, j=1,2, \cdots, m, \\
& \text { where } \tilde{R}_{j} \text { and } w_{1}^{*} \text { are as in }\left(\mathrm{F} 6^{\prime}\right) .
\end{aligned}
$$

Then, besides the periodic solution $x$ constructed in Theorem 4.4, the boundary value problems (1.1), (1.3) have another positive periodic solution $\tilde{x}$ with $r<$ $\|\tilde{x}+P\| \leq R$.

\section{REFERENCES}

[1] Carlos Alvarez and Alan C. Lazer, An application of topological degree to the periodic competing species problem, J. Austral. Math. Soc. Ser. B 28 (1986), no. 2, 202-219, DOI 10.1017/S0334270000005300. MR862570 (87k:34062)

[2] Jifeng Chu, Pedro J. Torres, and Meirong Zhang, Periodic solutions of second order nonautonomous singular dynamical systems, J. Differential Equations 239 (2007), no. 1, 196-212, DOI 10.1016/j.jde.2007.05.007. MR2341553(2008h:34029)

[3] Jifeng Chu and Juan J. Nieto, Impulsive periodic solutions of first-order singular differential equations, Bull. Lond. Math. Soc. 40 (2008), no. 1, 143-150, DOI 10.1112/blms/bdm110. MR2409187(2009c:34060)

[4] Meng Fan, Ke Wang, and Daqing Jiang, Existence and global attractivity of positive periodic solutions of periodic $n$-species Lotka-Volterra competition systems with several deviating arguments, Math. Biosci. 160 (1999), no. 1, 47-61, DOI 10.1016/S0025-5564(99)00022-X. MR.1704338 (2000f:92016)

[5] Xiao Han, Shuguan Ji, and Zhonghua Ma, On the existence and multiplicity of positive periodic solutions for first-order vector differential equation, J. Math. Anal. Appl. 329 (2007), no. 2, 977-986, DOI 10.1016/j.jmaa.2006.07.009. MR2296900(2007k:34054)

[6] Daqing Jiang, Jifeng Chu, and Meirong Zhang, Multiplicity of positive periodic solutions to superlinear repulsive singular equations, J. Differential Equations 211 (2005), no. 2, 282-302, DOI 10.1016/j.jde.2004.10.031. MR2125544(2005k:34087)

[7] V. Lakshmikantham, Periodic boundary value problems of first and second order differential equations, J. Appl. Math. Simulation 2 (1989), no. 3, 131-138. MR.1020149 (90j:34028)

[8] Eduardo Liz and J. J. Nieto, Periodic boundary value problems for a class of functional-differential equations, J. Math. Anal. Appl. 200 (1996), no. 3, 680-686, DOI 10.1006/jmaa.1996.0231. MR 1393108 (97k:34097)

[9] J. Mawhin, Topological degree and boundary value problems for nonlinear differential equations, Topological methods for ordinary differential equations (Montecatini Terme, 1991), Lecture Notes in Math., vol. 1537, Springer, Berlin, 1993, pp. 74-142, DOI 10.1007/BFb0085076. MR.1226930(94h:47121)

[10] Shiguo Peng, Positive solutions for first order periodic boundary value problem, Appl. Math. Comput. 158 (2004), no. 2, 345-351, DOI 10.1016/j.amc.2003.08.090. MR.2094624 (2005i:34029)

[11] Pedro J. Torres, Existence of one-signed periodic solutions of some second-order differential equations via a Krasnoselskii fixed point theorem, J. Differential Equations 190 (2003), no. 2, 643-662, DOI 10.1016/S0022-0396(02)00152-3. MR1970045(2003k:34082)

[12] Yonghui Xia and Maoan Han, New conditions on the existence and stability of periodic solution in Lotka-Volterra's population system, SIAM J. Appl. Math. 69 (2009), no. 6, 15801597, DOI 10.1137/070702485. MR2487162(2009k:34098)

[13] Huaiping Zhu, Sue Ann Campbell, and Gail S. K. Wolkowicz, Bifurcation analysis of a predator-prey system with nonmonotonic functional response, SIAM J. Appl. Math. 63 (2002), no. 2, 636-682 (electronic), DOI 10.1137/S0036139901397285. MR1951954 (2003m:34113)

School of Mathematical Sciences, Dalian University of Technology, Dalian, LiaonING 116024, PeOple's Republic of China

E-mail address: wangy891@gmail.com 